\title{
The Influence on Immune Cells by Aerobic Exercise and Detraining \\ Qiaozhen Yan
}

School of Wen Shan University, Wen Shan 63000, China

Yanqiaozh@163.com

Keywords: Aerobic exercise, Detraining, Immune cells

\begin{abstract}
With 14 college students as the research object, Were randomly divided into detraining group (group D) and aerobic exercise group (group A),D group stop training after 4 weeks' training, A group training eight weeks, to observe their effects on the immune system function. Found at the beginning of exercise, A group and D group all inhibition, restored gradually at the fourth week, group A is more obvious than group D. Detraining 2 weeks, each index has returned to normal, according to the experiment 2 weeks is the optimal time for detraining.
\end{abstract}

\section{Introduction}

Aerobic exercise is human body under the situation of physical exercise in which the oxygen supply fully, in the process of exercise, the human body equals the demand for oxygen and oxygen inhalation, oxygen to reach the equilibrium state, include some of low intensity, long time exercise, such as: walking, jogging, setting-up exercise, power bicycle, etc[1].The persistence of the long-term aerobic exercise can effectively improve cardiovascular and pulmonary function, increase the amount of $\mathrm{Hb}$ in the body, improve body resistance, anti-aging, enhance the working efficiency of the cerebral cortex, increase the consumption of fat, prevent arterial sclerosis, reduce the incidence of disease of heart head blood-vessel, etc. This article research on 14 freshman who study in Xi'an physical university, by aerobic endurance training, testing the body's immune cells changes in different movement period, provide aerobic exercise to improve the body quality, provides the theory basis for improving immunity in further, to deeply understand the effect of aerobic exercise to the body's immune system to lay the theoretical foundation.

\section{Research contents and methods}

Research object. 14 male students in grande one inhuman movement science in Xi'an physical university, mean age $19.5 \pm 0.7$ years, average height of $1.75 \pm 08 \mathrm{~m}$, an average of $64.40 \pm 3.35 \mathrm{~kg}$ weight, healthy and without any professional sports training, were randomly divided into having group (group D) and aerobic exercise group (group A).

Training arrangement. The students have aerobic exercise by treadmill( $(9 \mathrm{~km} \cdot \mathrm{h}-1$, grade 0 ) for 45 minutes, a total of eight weeks, the have aerobics exercise every other day.

Testing schedule. At 0, 4, 6, 8 weeks after the weekend aerobic exercise 1 hour take blood, every boy take venous blood $5 \mathrm{ml}$, separation of serum, standby testing relevant indicators of immune function.

Testing index. WBC count, GRAN count, GRAN\%,LYMPH count, LYMPH\%,MID count, MID\%.

Data processing. all the data using the mean and standard deviation $\bar{x} \pm \mathrm{s}$, by statistical software SPSS, significant differences $\mathrm{P}<0.005$.

\section{Experimental results}

Form table 1, the total number of in WBC, GRAN, LYMPH, MID in A group decreased obviously $(\mathrm{p}<0.01)$, compared the sixth week to the zero week GRAN have very significant difference $(\mathrm{p}<0.01)$, compared to the zero week, the WBC had significant difference $(\mathrm{p}<0.05)$, compared with the sixth week and the fourth week, the total number of WBC, LYMPH, MID 
significantly increased( $\mathrm{p}<0.01)$;Compared to the eighth week and the zero week, in addition to the total number of WBC has significant difference $(\mathrm{p}<0.05)$, and other indexes have no obvious change, compared the eighth week with the fourth week, the total number of WBC, LYMPH, MID significantly increased( $<<0.01)$; In group $\mathrm{D}$, compared the fourth week to the zero week, the total number of in WBC, GRAN, LYMPH, MID decreased obviously( $\mathrm{p}<0.01)$,compared the sixth week to the zero week, alough every indexs have changes, but have no significant difference; compared with the sixth week and the fourth week, the total number of WBC,GRAN, LYMPH, MID significantly increased( $\mathrm{p}<0.01)$; compared with the eighth week and the fourth week, the total number of WBC,GRAN, LYMPH, MID significantly increased( $\mathrm{p}<0.01)$.Compared to group A and Group D, in the fourth week and the eighth week, there have no significant difference, in the sixth week, WBC and LYMPH have significant difference $(\mathrm{p}<0.05)$, GRAN have a very significant difference $(\mathrm{p}<0.01)$.

Table 1 The change of immune cells in aerobic exercise and detraining

\begin{tabular}{lcccc}
\hline \multicolumn{5}{c}{ A 组 } \\
\hline \multicolumn{1}{c}{ (N=7) } & WO & W6 & W8 \\
& & & & \\
& & & & \\
& & & & \\
WBC (109/L) & $11.64 \pm 1.87$ & $4.68 \pm 0.89^{* *}$ & $8.96 \pm 2.54^{* \# \#}$ & $9.90 \pm 1.56^{* \# \#}$ \\
GARN (109/L) & $7.07 \pm 0.58$ & $3.65 \pm 0.80^{* *}$ & $9.09 \pm 1.31^{* *}$ & $9.54 \pm 1.47 \# \#$ \\
LYMPH (109/L) & $4.24 \pm 1.22$ & $1.27 \pm 0.28^{* *}$ & $3.78 \pm 0.36 \# \#$ & $4.19 \pm 0.30 \# \#$ \\
MID (109/L) & $0.94 \pm 0.12$ & $0.26 \pm 0.07^{* *}$ & $0.79 \pm 0.12 \# \#$ & $0.88 \pm 0.12 \# \#$ \\
& & & & \\
\hline
\end{tabular}

Table 2 The change of immune cells in aerobic exercise and detraining

\begin{tabular}{lccc}
\hline \multicolumn{5}{c}{ D 组 } \\
\cline { 2 - 4 } WO & W6 & & W8 \\
& & & \\
& & & \\
& & & \\
$6.97 \pm 1.58$ & $3.05 \pm 0.80^{* *}$ & $5.45 \pm 1.50 \# \#$ & $6.69 \pm 1.22 \# \#$ \\
$4.74 \pm 1.25$ & $1.37 \pm 0.28^{* *}$ & $4.23 \pm 0.40 \# \#$ & $4.28 \pm 0.36 \# \#$ \\
$0.96 \pm 0.42$ & $0.25 \pm 0.07^{* *}$ & $0.81 \pm 0.62 \# \#$ & $0.86 \pm 0.21 \# \#$ \\
& & & \\
\hline
\end{tabular}

\section{Analysis and discussion.}

White Blood cells(the WBC) is one of the cells in the Blood, are often called immune cells, when bacteria enter the body, White Blood cells can pass through the capillary wall by deformation, focus on bacteria invasion, surrounded the germs, phagocytosis. It has swallowed foreign body and the role of antibodies, healed the body injury damage ,he ability of resistance to pathogen invasion, immune ability to fight disease and so on. Lymphoid cells (lymphocyte) is a type of white blood cells, produced by the lymphoid organs, the body's immune response function of the main components. Lymphocyte percentage (LYMPH \%) belong to the white cell count, refers to the lymphocyte count and calculate the percentage of white blood cells. Neutrophils with deformation 
of the active movement and phagocytosis, play an important role in defense, the consuming object is given priority to with bacteria, in the blood of the nonspecific immune system plays a very important role. It plays an important role in defence besides the anti-infection, also can cause the infection of inflammation and participate in the parasitic infection caused by allergy, causing immune pathological damage. Cells in the middle (MID) including monocytes, eosinophils and basophils.

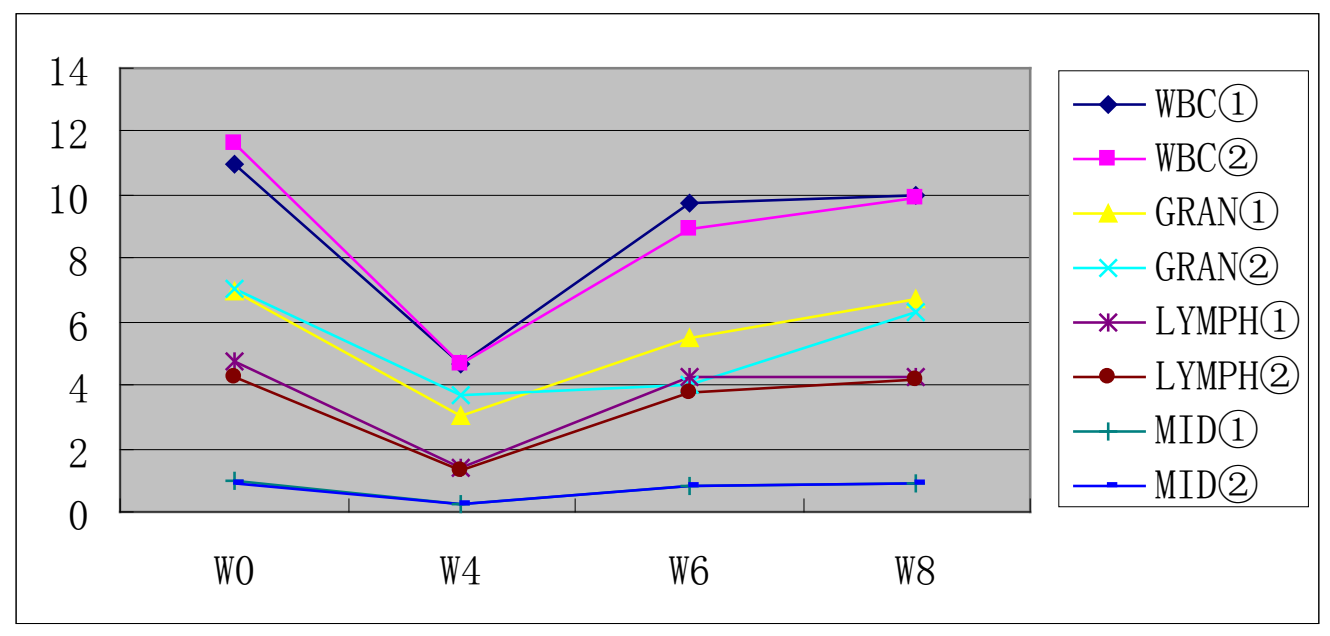

Fig1.The change of immune cells in group A and group D

From fig1, As a response to 8 weeks aerobic exercise training, the different immune cells showed different trends. At the beginning of the aerobics exercise, the WBC have a sharp drop in total, reached the lowest at the end of the aerobic exercise in the fourth week, which recovered in the next 4 weeks, at the eighth week, the total number of WBC is recovered the level before aerobics exercise. The change of GRAN and LYMPH are similar to WBC, just no so apparent. its began to recover in the fourth week. GRAN is restore slowly from the fourth week to the sixth week, they have basic recovery until the 8th week. LYMPH recovered to the level before aerobics exercise at the sixth week. Although the total number of MID fluctuate, but the change is not particularly evident.

TongHai [2]study the WBC changes on 60 male and a female college students before and after exercise, found that after exercise the total number of WBC, neutrophils and monocytes growth rate increases with longer duration of movement $(\mathrm{P}<0.05)$, while people of different training level of change is also different. Hessler [3] points out that the expression of neutrophils CD62Lreduced after motion, and subsequent recovery period for increased performance. Xiao-qiong Chen [4] found that the athletes in the movement when white blood cell count increased, while long-term training professional athletes won't appear the phenomenon of leukocytosis, in long-term endurance training, the degree of leukocytosis reduce. Ji-bin sha [5] don't think white blood cells of different subsets in at the same time, phase change was mainly affected by exercise intensity and exercise. Peake [6] etc. Through the study of the moderate intensity training of 10 male athletes , find that exercise can significantly increased number of neutrophils, after one hour after motion neutrophils continue to rise, $24 \mathrm{~h}$ after motion to return to the front of the movement. Saygin [7] by athletes compared with control group, such as volleyball players neutrophil number increased significantly.

In this experiment, LYMPH recover faster than in GRAN, possibly and its execution in the body's immune function. LYMPH undertake the specific immunity in the body, mainly against the invasion of specific antigen (such as viruses, bacteria, microbial pathogens) to identify and kill, so as to stress reaction of fading of movement, LYMPH of damaged tissue and necrotic muscle cells clean-up also end (process needs certain time), so the recovery faster. In the process of recovery, the recover of WBC is faster than A group, after stop movement, organization pool reduced demand for GRAN, break the GRAN's balance between rise and reduce in the cycle pool, in D group, the speed of GRAN migration to organization pool is slower than A group. And the promotion of the GRAN division, but the adjust factor of proliferation and promotion which promote it into the circulation pool still exist, so the cycle pool GRAN restore fast in the A group. Because the quantity 
of GRAN accounts for more than two-thirds of the total number of WBC, the WBC highly related, so it changes determines the change of the WBC, having set the WBC recovery speed is fast in the exercise group.

\section{Conclusion}

In aerobic exercise and detraining, immune cells immunosuppression at first, than recover gradually, to varying degrees of inhibition between different groups.

\section{References}

[1]Q.P.Huang: Journal of Beijing Sport University Journal of Beijing Sport University, Vol.12(2005), 28: 1649-1651. (In Chinese)

[2]H. Tong and W.Cui:Journal of Modern Laboratory Medicine,Vol.4(2007),22:64-65.(In Chinese)

[3]W.He and J.N.Chen:Chinese Journal of Tissue Engineering Research,Vol.10(2006),10:136-139. (In Chinese)

[4]X. Q.Chen:Heilongjiang Science and Technology Information, Vol.(2013),7:60-61.(In Chinese)

[5]J. B.Sha and L.Zhao:Journal of Tianjin University of Sport,Vol.3(1998),13:8-13.(In Chinese)

[6]Peake J M,Suzuki K, Wilson G,et a1.Exercise-induced muscle damage, plasma cytokines,and markers of neutrophll activation[J].Med Sci Sports Exerc,2005,37(5):737-745.

[7]Saygin K, Karacabey K,Ozmerdivenli R,et a1.Effect of chronic exercise oil immunoglobin, complement and leukocyte types in volleyball players and athletes[J]. Neuro Endocrino1 Lett, 2006,27(1-2):271-276. 